\title{
Lutte chimique contre le gui (Viscum album L.). Pénétration, transport, efficacité de deux herbicides phloème-mobiles (2,4-DB et glyphosate)
}

\author{
Françoise BAILLON ${ }^{* * * *(1)}$, A. CHAMEL ${ }^{*}$, A. FER ${ }^{* * *}$ et H. FROCHOT ${ }^{* * * *}$ \\ avec la collaboration technique de B. GAMBONNET * et M.C. MANZATO * \\ * Laboratoire de Biologie végétale, Département de Recherche fondamentale, \\ Centre d'Etudes nucléaires de Grenoble, 85 X 38041 Grenoble Cedex (France) \\ ** Laboratoire de Botanique et de Biologie végétale, \\ Université scientifique et médicale de Grenoble, Domaine Universitaire, \\ B.P. 68, 38402 Saint-Martin-d'Hères (France) \\ *** Laboratoire de Physiologie du Parasitisme végétal, Université de Nantes, \\ 2, rue de la Houssinière, 44072 Nantes (France). \\ **** I.N.R.A.-C.R.F., Laboratoire de Malherbologie, Station de Sylviculture, \\ Centre de Recherches de Nancy, Champenoux, 54280 Seichamps (France)
}

\begin{abstract}
Résumé
Une méthode de lutte chimique contre le gui des feuillus (Viscum album album, BALL) a été envisagée à l'aide de deux herbicides phloème-mobiles (2,4-DB et glyphosate) appliqués sur les feuilles du parasite.

Avant leur distribution dans le parasite, les herbicides doivent pénétrer à travers la cuticule des feuilles. L'influence de plusieurs paramètres (concentration, $\mathrm{pH}$, cires cuticulaires solubles) sur la fixation et la perméabilité cuticulaires des deux herbicides a été étudiée in vitro à l'aide de cuticules isolées. Leur distribution dans le parasite, fixé sur son hôte, a été suivie à trois stades caractéristiques du développement du gui. Elle présente des analogies avec celle des assimilats, notamment par le rôle de centre d'appel des jeunes pousses en croissance. Les herbicides ont été détectés en faible quantité dans le système endophytique du parasite six semaines après le traitement. Par contre aucune trace de phytotoxicité n'a été décelée dans l'hôte. Enfin, l'efficacité de ces deux herbicides, utilisés à $10 \mathrm{~g} / \mathrm{l}$ de 2,4-DB et $40 \mathrm{~g} / \mathrm{l}$ de glyphosate, a été vérifiée in situ.

Ces herbicides peuvent être utilisés efficacement et sans danger pour l'hôte, en application localisée sur les touffes de gui. Le stade de développement le plus intéressant pour le traitement semble être la fin du repos hivernal, au tout début du printemps, avant l'apparition des jeunes pousses du parasite.
\end{abstract}

Mots clés: Viscum album album BALL, hémiparasite, symptômes de phytotoxicité, fixation cuticulaire, perméabilité cuticulaire.

\section{Introduction}

Le gui, Viscum album L., est un hémiparasite chlorophyllien implanté sur de nombreux arbres et arbustes des régions tempérées. Trois sous-espèces peuvent être

(1) Actuellement : Laboratoirc de Biologic végétale, Division de Chimie, ED 5A, Centre Commun de Recherche, 21020 ISPRA (VA - Italie) 
différenciées suivant leur spécificité pour l'arbre-hôte : le gui du pin $(V$, a. austriacum, BALL), le gui du sapin ( $V$. a. abietis, BALL) et le gui des feuillus ( $V$. a. album, BALL), (VoN TuBEuF, 1923 ; BALL, 1964).

Le gui assure sa fixation sur l'hôte, sa multiplication végétative et son alimentation hydrique et minérale par l'intermédiaire de son système endophytique (fig. 1), ensemble de cordons corticaux et de suçoirs en connection directe avec les vaisseaux du bois de l'hôte. Par contre, il est autotrophe vis-à-vis du carbone (SEledzhanu et GalanFabian, 1961 ; Baillon et al., 1983).

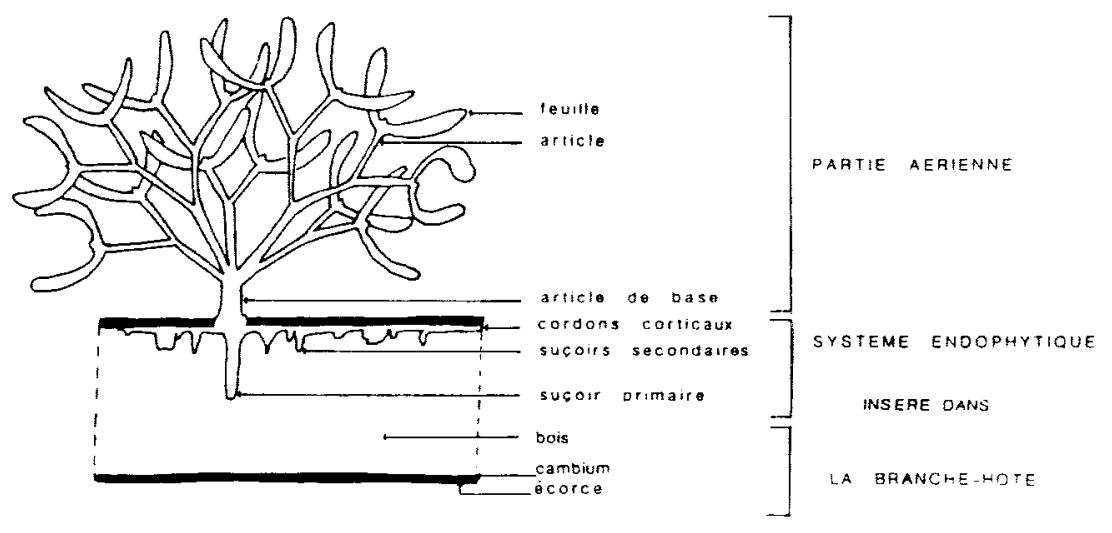

FiG. I

Morphologie du gui (Viscum album), d'après DEOM (198I).

Mistletoe anatomy (from DEom, 1981).

Le gui provoque des dégâts importants sur de nombreuses espèces ligneuses forestières et fruitières : détérioration du bois, pertes de croissance, affaiblissement allant parfois jusqu’à la mort de l'arbre (Frochot et SAlle, 1980). La destruction de la seule partie aérienne du parasite est suivie de l'apparition de rejets. Aussi, une lutte efficace doit détruire ou inhiber le système endophytique. Cet objectif peut être atteint grâce à l'emploi d'herbicides phloème-mobiles - c'est-à-dire transportés par la sève élaborée - appliqués directement sur les feuilles du parasite (Dei.ABraze et LANiER, 1972 ; Frochot et Delabraze, 1979 ; Frochot et al., 1983 ; Maréchal, 1983).

Dans le but d'obtenir une meilleure connaissance des phénomènes, une étude physiologique a été réalisée à l'aide de deux herbicides phloème-mobiles, le 2,4-DB ou acide 2,4-dichloro-phénoxy-butyrique et le glyphosate ou $\mathrm{N}$-phosphono-methyl-glycine. D'une part, nous avons cherché à préciser in vitro le comportement de ces matières actives vis-à-vis de cuticules isolées de feuilles de gui. D'autre part, nous avons suivi leur distribution dans le parasite à trois stades du développement phénologique du gui et leur éventuel passage dans l'hôte. Enfin, nous avons vérifié l'efficacité globale sur le gui des deux herbicides testés. 


\section{Matériel et méthodes}

\subsection{Pénétration cuticulaire}

Rappel de la structure de la cuticule des plantes.

La feuille des plantes supérieures est recouverte d'une cuticule dont l'épaisseur varie entre 0,5 et $15 \mu \mathrm{m}$ suivant les espèces. Cette cuticule est constituée de la surface vers l'intérieur (Chamel, 1986) par : ques) :

- les cires épicuticulaires (mélange de classes de composés aliphatiques et cycli-

- la cuticule sensu-stricto, constituée de cutine (polyesters d'acides gras en C16 et C18, hydroxylés et époxylés) et de cires intracuticulaires;

- les couches cuticulaires, composées aussi de cutine et de cires associées ou non à du matériel fibrillaire provenant de la paroi cellulaire épidermique externe.

La cuticule de feuille de gui (fig. 2) a une épaisseur d'environ $10 \mu \mathrm{m}$. Elle présente de nombreuses empreintes stomatiques, en nombre égal sur les deux faces de la feuille $\left(5500 / \mathrm{cm}^{2}\right)$. Sur les cuticules isolées, les ostioles apparaissent fermés. En microscopie électronique à balayage, les cires épicuticulaires se présentent sous forme de bâtonnets isolés.

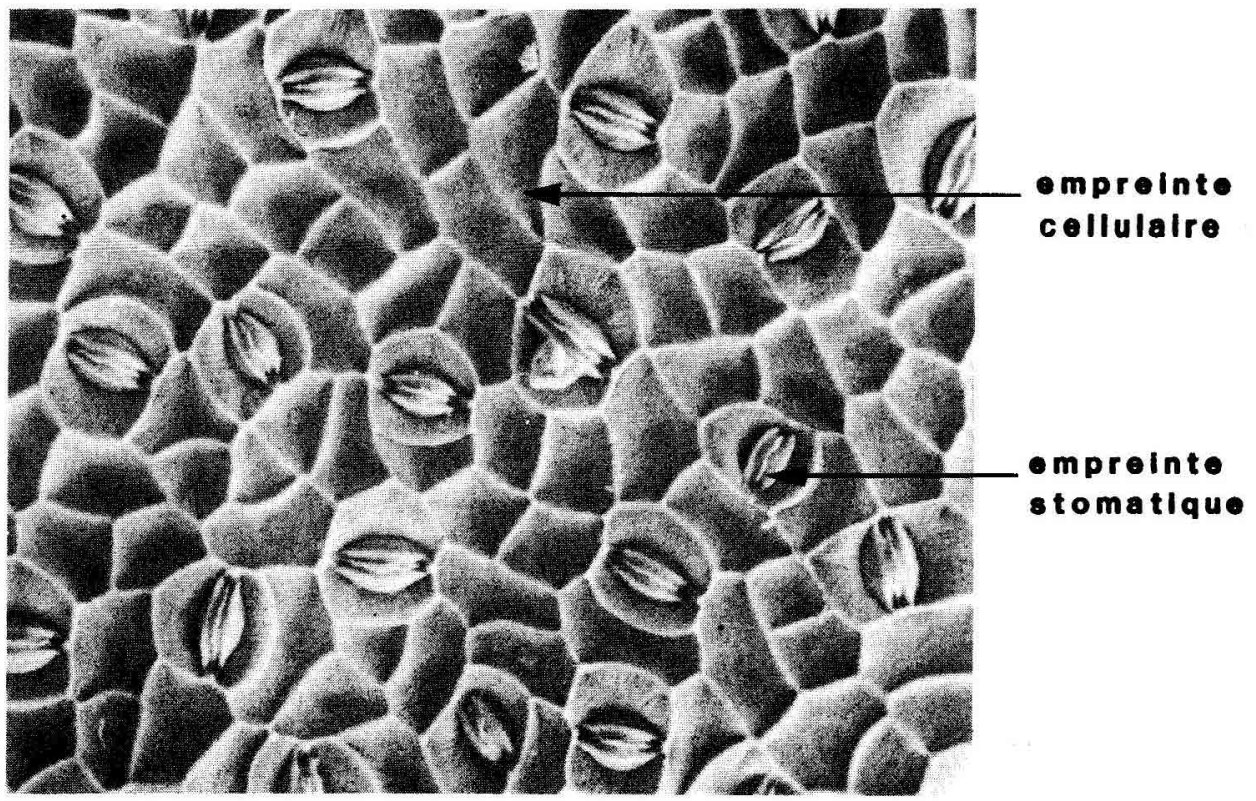

FIG. 2

Face interne de la cuticule isolée de feuille de gui (Viscum album) avec les empreintes stomatiques. (Microscopie êlectronique à balayage, $1 \mathrm{~cm}=62,5 \mu \mathrm{m}$ ).

Internal side of an isolated cuticle of mistletoe leaf; stomatal prints. 
La pénétration cuticulaire des herbicides, 2,4-DB et glyphosate, utilisés sous forme de matière active pure marquée au carbone 14, est étudiée à l'aide de cuticules isolées. Elles sont obtenues à partir de disques foliaires (diamètre égal à $1 \mathrm{~cm}$ ) découpés dans des feuilles d'un an, et sont immergées sous agitation dans une solution enzymatique appropriée (Chamel et Bougre, 1977). L'étude de l'influence des cires cuticulaires sur la pénétration des herbicides nécessite de disposer également de cuticules « décirées». Elles sont préparées par immersion des cuticules isolées dans le chloroforme (BaILlon, 1985). Les cires solubles représentent 23 p. 100 de la masse sèche des cuticules.

Deux paramètres caractérisent la pénétration cuticulaire : la fixation et la perméabilité. Pour chaque herbicide est étudiée l'influence sur ces deux paramètres de la concentration, du $\mathrm{pH}$ et des cires cuticulaires. Les conditions particulières à l'étude de chaque facteur sont indiquées dans les tableaux 1 et 2 .

\subsection{Fixation cuticulaire (Chamel et Bougie, 1977 ; Baillon, 1985)}

Des lots de 25 disques cuticulaires sont maintenus immergés à $25^{\circ} \mathrm{C}$, sous agitation constante, pendant 24 heures, dans une solution contenant l'herbicide marqué. A la fin de la période d'immersion, les disques cuticulaires sont lavés $(10 \mathrm{~min}$, soit dans $10 \mathrm{ml}$ d'éthanol 10 p. 100 avec le 2,4-DB, soit dans $10 \mathrm{ml}$ d'eau dans le cas du glyphosate). Chaque disque est ensuite solubilisé dans $1 \mathrm{ml}$ de liquide solubilisateur (lumasolve, LUMAC) et la radioactivité est mesurée par scintillation liquide.

\subsection{Perméabilité cuticulaire (Riederer et Schonherr, 1985 ; BAILlon, 1985)}

Elle a été mesurée à l'aide d'un dispositif composé de deux compartiments en verre pouvant communiquer entre eux. Pour l'expérience, un disque cuticulaire, choisi sans défaut après observation au microscope optique, est inséré dans l'ouverture entre les deux compartiments. Ces derniers contiennent chacun $10 \mathrm{ml}$ d'une même solution tampon. La surface de la cuticule qui est en contact avec la solution est $0,385 \mathrm{~cm}^{2}$.

Au début de l'expérience, l'herbicide marqué est introduit dans le compartiment situé du côté de la face externe de la cuticule, appelé compartiment donneur. Des prélèvements (fractions aliquotes de $100 \mu \mathrm{l}$ ) sont effectués dans l'autre compartiment, appelé receveur, après $0,1,3,6,24,48,72$ et 144 heures. Leur radioactivité est mesurée par scintillation liquide, permettant ainsi de connaitre la quantité d'herbicide ayant traversé la cuticule. Après chaque prélèvement, un volume de solution tampon égal au volume prélevé, est ajouté dans le compartiment receveur, pour qu'il n'y ait pas de différence de pression hydrostatique entre les deux compartiments. La perméabilité est exprimée par le coefficient de perméabilité $\mathrm{P}$ :

$$
\mathrm{P}=\mathrm{dn} / \mathrm{dt} \times 1 / \mathrm{C}(\mathrm{cm} / \mathrm{s})
$$

où $\mathrm{C}:$ concentration de l'herbicide dans le compartiment donneur

$$
\text { (DPM } / \mathrm{cm}^{3} \text { - DPM : Désintégrations Par Minute) }
$$

$\mathrm{dn} / \mathrm{dt}$ : vitesse de pénétration de l'herbicide à travers la cuticule $\left(\mathrm{DPM} / \mathrm{cm}^{2} / \mathrm{s}\right)$. 


\subsection{Distribution dans le parasite}

Trois stades de développement du gui ont été choisis pour l'application des 2 herbicides : avant (stade I), au début (stade II) et à la fín (stade III) du développement des jeunes pousses du parasite. L'expérimentation a été conduite à l'aide de systèmes simplifiés hôte-parasite, constitués chacun d'une branche de peuplier (Populus sp.) parasitée par le gui et bouturée depuis un an (Frochot et WERLHEN, à paraître). A chaque ensemble hôte-parasite correspond une modalité expérimentale, définie par un herbicide et un stade d'application. L'ensemble des six modalités ( 2 herbicides, 3 stades) a été répété deux fois, réparti sur deux années consécutives. Chaque ensemble hôte-parasite a été laissé en pot ( $1^{\mathrm{re}}$ année) ou placé en pleine terre ( $2^{\mathrm{e}}$ année), à l'abri de la pluie et abondamment irrigué.

La distribution du 2,4-DB et du glyphosate dans le parasite a été étudiée à l'aide de matière active pure marquée au carbone 14. Les herbicides en solution ont été appliqués sur des feuilles adultes du parasite dont le limbe a été préalablement piqué à l'aide d'une aiguille ( 30 piqûres rêparties de part et d'autre de la nervure centrale), afin de supprimer la barrière cuticulaire. Les caractéristiques exactes des solutions herbicides employées sont indiquées dans la légende de la figure 3. La quantité totale déposée est la même pour chaque herbicide à $10 \mu \mathrm{g}$ près. Elle est très en dessous des quantités ayant déjà entrainé la destruction de la partie aérienne du parasite. Vingt-quatre heures après l'application de la solution herbicide, un dépôt d'eau distillée (dans le cas du glyphosate) ou d'éthanol 10 p. 100 (dans le cas du 2,4-DB) a été effectué pour favoriser la pénétration.

En fin d'expérience ( 6 semaines après chaque traitement), la radioactivité a été recherchée dans l'appareil aérien du gui, dans son système endophytique et dans la branche-hôte. Les feuilles traitées ont été prélevées, puis lavées ( 3 fois 2 minutes dans de l'eau distillée ou de l'éthanol 10 p. 100) afin de déterminer la quantité d'herbicide marqué qui n'a pas été absorbée. Les différentes parties du plant de gui et les coupes transversales de tige de peuplier, prélevées au point d'implantation du parasite, ont été autoradiographiées (durée d'exposition 2 mois). Les résultats ont été ensuite quantifiés. Pour cela, la combustion des échantillons (Oxidizer - Packard Instrument) a permis de récupérer le ${ }^{14} \mathrm{C}$ de l'herbicide par piégeage $\mathrm{du} \mathrm{CO}_{2}$ dégagé, et la radioactivité a été mesurée par scintillation liquide.

\subsection{Essai d'efficacité des deux herbicides}

Un essai a été réalisé dans le massif du Vercors, dans le sud-est de la France (altitude $850 \mathrm{~m}$ ), sur des plants de gui en place sur un pommier (Pirus Malus L.). Les herbicides ont été utilisés sous forme de produits commerciaux : Embutone $(300 \mathrm{~g} / 1 \mathrm{de}$ 2,4-DB sels de sodium et de potassium) et Roundup $(360 \mathrm{~g} / 1$ de glyphosate sel d'isopropylamine). Ils ont été employés en solution aqueuse à 10 et $40 \mathrm{~g}$ de matière active par litre. Les solutions ont été appliquées sur la totalité de chaque touffe de gui par pulvérisation à «goutte tombante », c'est-à-dire jusqu'à formation d'une goutte à l'extrémité des feuilles. Les traitements ont été effectués sur 8 touffes de gui de taille et de situation comparable, établies sur les grosses branches d'un même arbre. Ils ont été répétés deux fois pour chaque herbicide à chaque concentration. Les pulvérisations ont été effectuées fin mars 1984, dans des conditions météorologiques satisfaisantes pour l'application des produits (soleil et absence de vent). Le gui était au stade « fin de floraison », avant l'apparition des nouvelles pousses et le pommier n'avait pas encore de feuilles. 


\section{Résultats}

\subsection{Pénétration cuticulaire}

\subsection{Fixation cuticulaire (tab!. 1)}

Pour les deux herbicides étudiés, la fixation cuticulaire est une fonction linéaire de la concentration (dans les limites fixées - tabl. la et d). D'autre part, les quantités de 2,4-DB fixées sont beaucoup plus élevées que celles de glyphosate (environ 10 fois). Ceci est à mettre en relation avec les caractères respectivement lipophile et hydrophile des deux molécules.

\section{TABLEAU 1}

Fixation sur des cuticules isolées de feuille de gui (Fix. cut.) du 2,4-DB et du glyphosate. Influence de la concentration, du pH et des cires cuticulaires solubles ${ }^{(*)}$.

2.4-DB and glyphosate sorption (Fix. cut.) on isolated cuticles of mistletoe leaf. Influence of concentration. $\mathrm{pH}$, and soluble cuticular waxes.

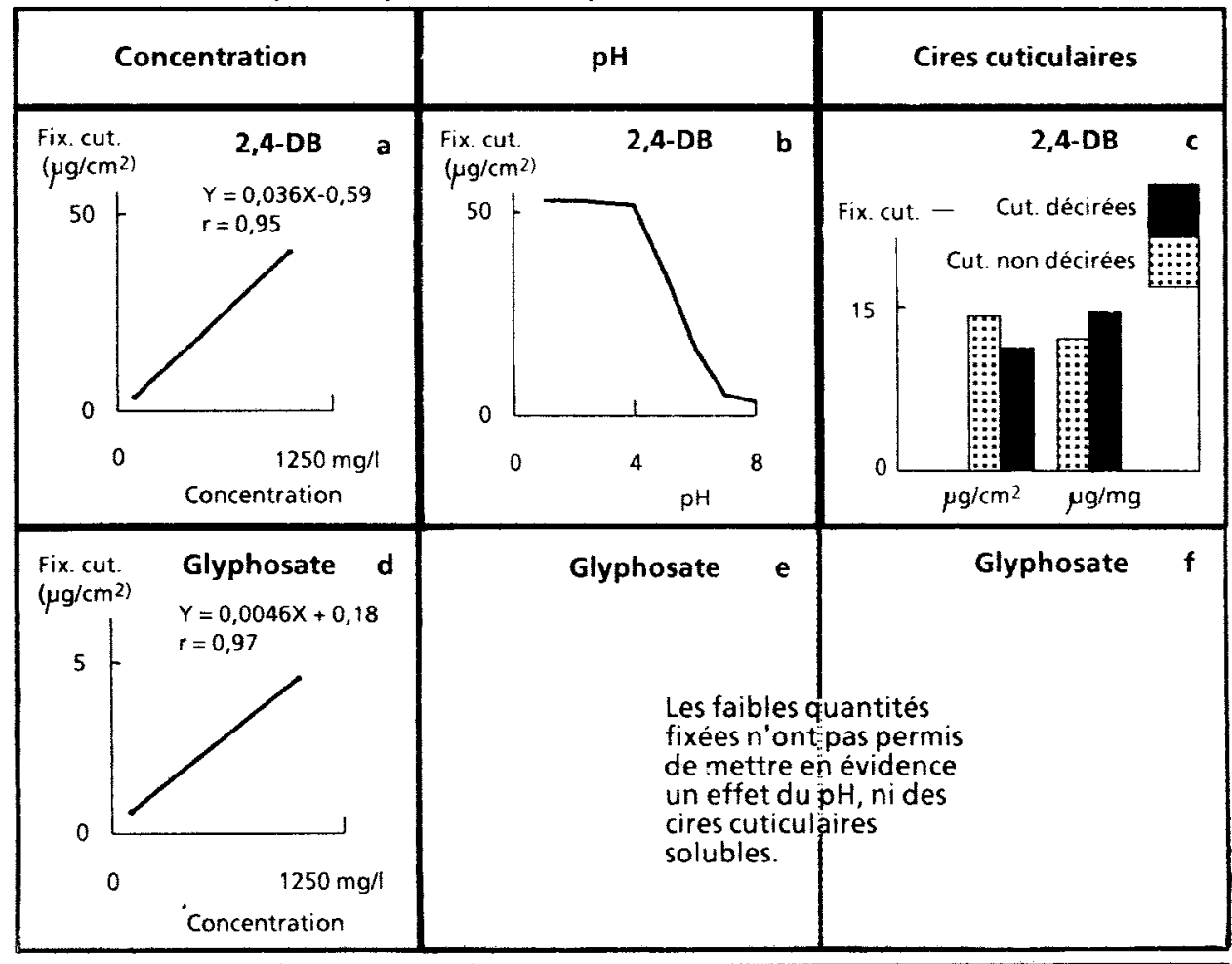

(*) Conditions d'expétience :

a $-0.2 \mu \mathrm{Ci} / \mathrm{ml}: \mathrm{pH}=4.4$; Ethanol $35 \mathrm{p} .100$

b $-\mathrm{C}=10^{-3} \mathrm{M} ; 1 \mu \mathrm{Ci} / \mathrm{ml}$; Ethanol $10 \mathrm{p} .1(0)$

c $-\mathrm{C}=10 \mathrm{M} ; 0,2 \mu \mathrm{Ci} / \mathrm{ml} ; \mathrm{pH}=4,5 ;$ Ethanol $10 \mathrm{p} .100$.

$\mathrm{d}-0.1 \mu \mathrm{Ci} / \mathrm{ml} ; \mathrm{pH}=3$.

c. $\mathrm{C}=10^{3} \mathrm{M} ; 0.1 \mu \mathrm{Ci} / \mathrm{ml}$.

f $-\mathrm{C}=10^{-3} \mathrm{M} ; 0.1 \mu \mathrm{Cl} / \mathrm{ml} ; \mathrm{pH}=3$. 
La fixation cuticulaire du 2,4-DB est plus élevée à pH faible. Elle décroît ensuite très rapidement pour des valeurs de $\mathrm{pH}$ proches de la constante de dissociation de l'herbicide, exprimée par son pKa (tabl. 1b).

La quantité de 2,4-DB fixée est plus importante avec les cuticules non décirées si elle est exprimée par unité de surface, et avec les cuticules décirées si elle est exprimée par unité de poids (tabl. Ic - valeurs significativement différentes pour $p=0,05$ ). Ainsi la cutine présente une affinité plus grande que les cires à l'égard du 2,4-DB. Celles-ci en retiennent environ 15 p. 100.

Dans le cas du glyphosate, les faibles quantités fixées n'ont pas permis de mettre en évidence un effet du $\mathrm{pH}$, ni des cires cuticulaires solubles.

\subsection{Perméabilité cuticulaire (tabl. 2)}

Il faut tout d'abord noter la grande variabilité des résultats obtenus avec différentes cuticules issues d'un même lot de feuilles ou de lots différents (tabl. $2-\operatorname{lot} 1$ : cuticules 1 à 5 ; lot 2 : cuticules 6 à 8 ). De plus, même si les quantités d'herbicide passant du compartiment donneur au compartiment receveur sont faibles $(0,5$ à 5 p. 100 de la quantité initialement fournie), il apparaît que la cuticule de feuille de gui est plus perméable au 2,4-DB qu'au glyphosate.

Dans une expérience parallèle, nous avons noté qu'à un pH donné, le coefficient de perméabilité ne dépend pas de la concentration de la solution herbicide (BAlllon, 1985). La quantité d'herbicide traversant la cuticule augmente donc linéairement avec la concentration.

\section{TABLEAU 2}

Perméabilité au 2,4-DB et au glyphosate de cuticules isolées de feuille de gui, exprimée par le coefficient de perméabilité $P(\mathrm{~cm} / \mathrm{s})$. Influence du $\mathrm{pH}$ et des cires cuticulaires solubles ${ }^{(*)}$

Permeability (permeability coefficient $P(\mathrm{~cm} / \mathrm{s})$ ) of isolated cuticles of mistletoe leaf to 2.4-DB and glyphosate. Influence of concentration, $p H$, and soluble cuticular waxes.

\begin{tabular}{|c|c|c|c|c|}
\hline \multirow{2}{*}{$\mathrm{P}(\mathrm{cm} / \mathrm{s})$} & \multirow{2}{*}{$N^{\prime \prime}$ Cut. } & \multicolumn{2}{|c|}{ Cuticules non décirées } & \multirow{2}{*}{$\begin{array}{c}\text { Cuticules décirées } \\
\mathrm{pH}=8\end{array}$} \\
\hline & & $\mathrm{pH}=3$ & $\mathrm{pH}=8$ & \\
\hline \multirow[t]{2}{*}{ 2.4-DB } & $\begin{array}{l}1 \\
2 \\
3\end{array}$ & $\begin{array}{l}2,96310^{-6} \\
3,18410^{-6} \\
1,17110^{-6}\end{array}$ & $\begin{array}{l}0,35510^{-6} \\
0,18010^{-6} \\
0,19910^{-6}\end{array}$ & $\begin{array}{l}1,02110^{-6} \\
5,38310^{-6} \\
2,40410^{-6}\end{array}$ \\
\hline & $\mathrm{N}^{\circ}$ Cut. & $\mathrm{pH}=3$ & $\mathrm{pH}=8$ & $\mathrm{pH}=3$ \\
\hline Glyphosate & $\begin{array}{l}4 \\
5 \\
6 \\
7 \\
8\end{array}$ & $\begin{array}{lll}3,83 & 10^{-7} \\
3,52 & 10^{-7} \\
0,37 & 10^{-7} \\
0,41 & 10^{-7} \\
0,40 & 10^{-7}\end{array}$ & $\begin{array}{ll}1,92 & 10^{-7} \\
0,69 & 10^{-7} \\
0,132 & 10^{-7}\end{array}$ & $\begin{array}{l}1,0810^{7} \\
1,1510^{-7}\end{array}$ \\
\hline
\end{tabular}

( $\left.{ }^{*}\right)$ Conditions d'expérience :

- $[2,4-\mathrm{DB}]=10^{-4} \mathrm{M} ; 0,5 \mu \mathrm{Ci} / \mathrm{ml} ;$ Ethanol 1 p. 100 .

- [glyphosate] $=10^{-4} \mathrm{M} ; 0,1 \mu \mathrm{Ci} / \mathrm{ml}$.

- $\mathrm{pH}=3:$ Tampon SADH $10 \mathrm{mM}$.

- $\mathrm{pH}=8$ : Tampon HEPES $10 \mathrm{mM}$

$\mathrm{N}^{\circ}$ Cut. : chaque numéro correspond à une cuticule différente extraite de deux lots de feuilles de gui (lot $1=$ cuticules 1 à $5: \operatorname{lot} 2=$ cuticules 6 à 8 ). 
Pour une même cuticule, les valeurs du coefficient de perméabilité à $\mathrm{pH}=3$ sont toujours plus élevées que celles obtenues à $\mathrm{pH}=8$ (tabl. 2) : environ 10 fois supérieures dans le cas du 2,4-DB et 2 à 5 fois dans celui du glyphosate. La courbe présentant la perméabilité cuticulaire au 2,4-DB en fonction du $\mathrm{pH}$ a un point d'inflexion pour une valeur de pH proche du pKa de l'herbicide (BaIllon, 1985).

L'extraction des cires solubles augmente la perméabilité cuticulaire des herbicides d'un mème ordre de grandeur qu'une variation de $\mathrm{pH}$ entre 8 et 3 (tabl. 2).

\subsection{Distribution dans le parasite}

Les résultats des deux répétitions sont tout à fait comparables, c'est pourquoi un seul autoradiogramme sera présenté par herbicide à chaque stade considéré.

Dans le cas du 2,4-DB (fig. 3A), au stade I la radioactivité se répartit dans l'ensemble des jeunes pousses (apparues en fin d'expérience), proches ou éloignées des feuilles traitées, ainsi que dans les articles qui les portent et dans l'article basal. Au stade II, la radioactivité n'apparaît que dans les feuilles les plus proches des feuilles traitées. Au stade III, la radioactivité semble moins exportée que dans le cas précédent : elle se limite à des traces dans les jeunes pousses et les articles situés immédiatement en dessous des feuilles traitées. A chaque stade, la radioactivité est décelable à l'état de trace dans le système endophytique.

Dans le cas du glyphosate (fig. 3B), au stade I, la radioactivité a été distribuée dans l'ensemble du parasite, mais les parties les plus proches des feuilles traitées apparaissent plus marquées. Des traces sont décelables dans l'article de base et dans le système endophytique du parasite. Au stade II, la radioactivité est présente principalement dans les jeunes pousses proches ou éloignées des feuilles traitées. Le système endophytique est à peine visible. Au stade III, la radioactivité est présente dans les jeunes pousses les plus proches des feuilles traitées, dans les articles situés immédiatement à leur base et de façon très nette dans le système endophytique. Elle est absente du reste du parasite.

Enfin, il est important de noter qu'aucune trace de radioactivité n'a jamais été décelée dans la branche-hôte.

Les résultats quantitatifs après lavage des feuilles traitées montrent une augmentation de la pénétration foliaire en fonction du stade de traitement. En effet, pour le 2,4-DB, 54 p. 100 de la radioactivité sont absorbés au stade I contre 97 p. 100 au stade III, pour le glyphosate 60 p. 100 contre 90 p. 100 . Dans ces essais, la pénétration foliaire était favorisée par les piqûres effectuées sur le limbe des feuilles traitées. Ceci explique les valeurs élevées obtenues avec des matières actives pures. En fait, la pénétration foliaire était seulement de 35 p. 100 au stade I lorsque le glyphosate était appliqué, dans les mêmes conditions, sur des feuilles intactes. Le bilan complet de la radioactivité (lavage + combustion des échantillons) indique que plus de 90 p. 100 de la quantité déposée initialement est récupérée. Il n'y a donc pas métabolisation complète des herbicides au cours des six semaines d'expérience.

Les résultats quantitatifs de la distribution de la radioactivité dans le parasite sont donnés dans le tableau 3. L'exportation de la radioactivité hors des feuilles traitées est plus importante pour les applications effectuées au début du développement (stade I). Pour le 2,4-DB, elle représente 26,3 p. 100 de la radioactivité totale du parasite. Elle n'est plus que de 1 p. 100 par la suite. Pour le glyphosate, elle passe de 21,4 p. 100 au 


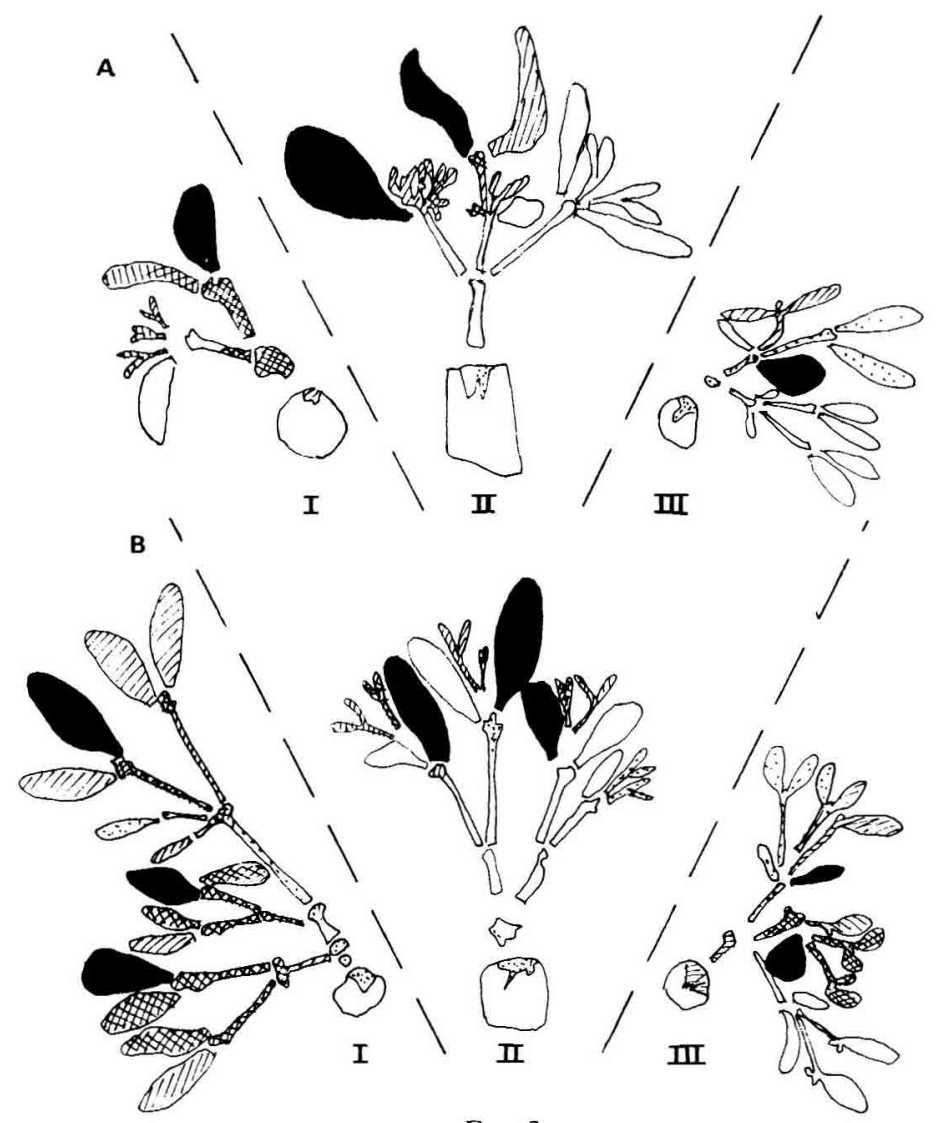

Fig. 3

Schéma de la distribution de la radioactivité chez le gui (Viscum album),

six semaines après le dépôt foliaire de 2,4-DB (A) et de glyphosate (B) marqués au carbone 14. Applications ${ }^{(*)}$ effectuées:

I- Avant le développement des jeunes pousses.

II - Au début du développement des jeunes pousses.

III - A la fin du développement des jeunes pousses.

Scheme of the radioactivity distribution in mistletoe six weeks after the foliar applications of ${ }^{14} \mathrm{C}$ labelled 2.4-DB $(A)$ and glyphosate $(B)$.

$$
\text { Herbicides applied: }
$$

I - Before the young shoot development.

II - at the beginning of the young shoot development.

III - at the end of the young shoot development.

Feuilles traitées / Treated leaves
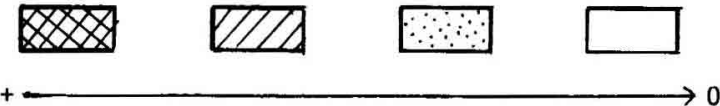

Radioactivité exportée hors des feuilles traitées/Radioactivity exported from the treated leaves

$\left({ }^{*}\right)$ Conditions d'expérience :

$\mathrm{A}-[2,4-\mathrm{DB}]=910^{-3} \mathrm{M}(450 \mu \mathrm{g}$ par plant de gui) $; 50 \mu \mathrm{Ci} / \mathrm{ml}$; Ethanol $10 \mathrm{p} .100$.

2 feuilles traitées par plant de gui.

B - [glyphosate] $=5,210^{-3} \mathrm{M}(440 \mu \mathrm{g}$ par plant de gui) $; 10 \mu \mathrm{Ci} / \mathrm{ml}$.

5 feuilles traitées par plant de gui. 
TABLEAU 3

Distribution dans le gui du ${ }^{14} \mathrm{C}-2,4-D B$ et du ${ }^{14} \mathrm{C}$-glyphosate (GLYP.) appliqués ${ }^{(*)}$ à trois stades de développement du parasite: avant, au début, à la fin du développement des jeunes pousses (stades I, II, III).

$P .100$ de la radioactivité totale présente dans la partie aérienne du parasite.

Distribution in mistletoe of ${ }^{14} \mathrm{C}-2.4-\mathrm{DB}$ and ${ }^{14} \mathrm{C}$-glyphosate applied at three stages of the parasite development : before, at the beginning, at the end of the young shoot development (stages I, II, III). P. 100 of the total radioactivity of the parasite aerial part.

\begin{tabular}{l|c|c|c|c|c|c}
\hline \hline \multirow{2}{*}{$\begin{array}{c}\text { Partie aérienne du plant de gui, } \\
\text { divisée en différents groupes } \\
\text { d'échantillons }\end{array}$} & \multicolumn{2}{|c|}{ Stade I } & \multicolumn{2}{c|}{ Stade II } & \multicolumn{2}{c}{ Stade III } \\
\cline { 2 - 7 } & $2,4-\mathrm{DB}$ & GLYP. & $2,4-\mathrm{DB}$ & GLYP. & $2,4-\mathrm{DB}$ & GLYP. \\
\hline Feuilles traitées & 73,75 & 78,62 & 98,84 & 98,84 & 99,20 & 96,21 \\
\hline $\begin{array}{l}\text { Feuilles opposées aux feuilles trai- } \\
\text { tées }\end{array}$ & 1,59 & 1,88 & $(* *)$ & 0,14 & 0,02 & 0,05 \\
\hline $\begin{array}{l}\text { Jeunes pousses les plus proches des } \\
\text { feuilles traitées }\end{array}$ & 19,30 & $(* *)$ & 0,54 & $\mathbf{0 , 6 2}$ & 0,30 & 2,60 \\
\hline Articles portant les feuilles traitées & 3,50 & 9,67 & 0,35 & $\mathbf{0 , 2 5}$ & 0,32 & 0,89 \\
\hline Reste du plant de gui & 1,86 & 9,83 & 0,27 & $\mathbf{0 , 1 6}$ & 0,17 & 0,25 \\
\hline
\end{tabular}

(*) Conditions d'expérience : voir fig. 3 . libéré.

Mesure de la radioactivité par scintillation liquide après combustion des échantillons et piégeage du ${ }^{\mathrm{f}} \mathrm{CO}_{2}$

(**) Absence du groupe d'échantillons correspondant.

\section{TABLEAU 4}

Evolution des symptômes observés sur le gui, parasite du pommier, après traitement ${ }^{(*)}$ par l'Embutone $(300 \mathrm{~g} / \mathrm{l}$ de 2,4-DB) et le Roundup $(360 \mathrm{~g} / \mathrm{l}$ de glyphosate).

Damage observations on mistletoe, growing on apple tree, after treatments with Embutone $(300 \mathrm{~g} / \mathrm{l} \mathrm{2.4-DB})$ and Roundup $(360 \mathrm{~g} / \mathrm{l}$ glyphosate).

\begin{tabular}{|c|c|c|c|c|}
\hline Spécialités commerciales & \multicolumn{2}{|c|}{ Embutone } & \multicolumn{2}{|c|}{ Roundup } \\
\hline Concentration de la solution & \multicolumn{2}{|c|}{$2,4-\mathrm{DB}$} & \multicolumn{2}{|c|}{ Glyphosate } \\
\hline Délai d'apparition des symptômes & $40 \mathrm{~g} / 1$ & $10 \mathrm{~g} / 1$ & $40 \mathrm{~g} / 1$ & $10 \mathrm{~g} / 1$ \\
\hline Nécroses sur les feuilles & 2 mois & $2-4$ mois & 2 mois & non \\
\hline Chute des feuilles & 2 mois & $2-4$ mois & 2 mois & 4 mois \\
\hline Altération du développement des jeunes pousses & 2 mois & $2-4$ mois & 2 mois & 2 mois \\
\hline $\begin{array}{l}\text { Desséchement complet du parasite dont la char- } \\
\text { pente reste en place }\end{array}$ & 4 mois & 6 mois & non & non \\
\hline Chute des articles, sauf à la base. Brunissement & non & non & 6 mois & 4 mois \\
\hline Repousses, mais avec altération de la croissance & non & non & non & $6-18$ mois \\
\hline
\end{tabular}

$\left(^{*}\right)$ Traitements effectués en mars 1984 , sur deux touffes de gui pour chaque herbicide à chaque concentration. 
stade I à 1 et 4 p. 100 aux autres stades. L'exportation préférentielle de la radioactivité vers les jeunes pousses, déjà mise en évidence par autoradiographie, est confirmée par les données quantitatives: c'est particulièrement net au stade I dans le cas du 2,4-DB, où 78 p. 100 de la radioactivité totale exportée se retrouve dans les jeunes pousses. Ce résultat n'a pu être vérifié avec le glyphosate du fait que les jeunes pousses n'étaient pas encore apparues à ce stade.

\subsection{Essai d'efficacité des deux herbicides}

L'ensemble des résultats obtenus a été résumé dans le tableau 4 . Les différents symptômes observés, ainsi que leur délai d'apparition, sont précisés dans chaque cas. Il faut observer la lenteur de réponse du parasite aux différents traitements herbicides. Les premiers symptômes apparaissent plus de deux mois après les traitements. Pour une efficacité maximum visible, il faut attendre 4 à 6 mois. Le gui présente une réponse différente aux deux herbicides; d'une part, la concentration minimale efficace est beaucoup plus faible pour le $2,4-\mathrm{DB}$ que pour le glyphosate ( 10 contre $40 \mathrm{~g}$ de matière active par litre), d'autre part, des symptômes particuliers caractérisent chaque herbicide (tabl. 4) : charpente desséchée restant en place dans le cas du 2,4-DB, altération et chute progressive des articles supérieurs, alors que les articles de base restent en place (ni secs, ni verts) dans le cas du glyphosate.

Il est en outre important de noter qu'aucune trace de phytotoxicité n'a été décelée sur l'arbre-hôte dans les conditions de notre essai.

\section{Discussion}

\subsection{Pénétration cuticulaire}

La grande variabilité intraspécifique observée a déjà été rapportée par plusieurs auteurs dans le cas de la pénétration d'ions minéraux et de molécules organiques (Chamel et Gambonnet, 1982 ; Riederer et Schonherr, 1985 ; Chamel, 1986). Ceci suggère une grande hétérogénéité dans la structure et l'arrangement des différents constituants de la cuticule.

Les résultats de la fixation et de la perméabilité cuticulaires sont à rapprocher. Pour les deux herbicides étudiés, la pénétration cuticulaire est une fonction linéaire de la concentration. Pour le 2,4-DB, herbicide type acide organique faible, la pénétration (fixation et perméabilité) est plus élevée à $\mathrm{pH}$ faible quand l'herbicide est sous forme non dissociée (R-COOH). Son affinité est alors plus forte pour les lipides, donc pour la cuticule. A pH élevé, il est sous forme dissociée $\left(\mathrm{R}-\mathrm{COO}^{-}\right)$, et son affinité est plus forte pour le milieu aqueux. Ces résultats sont tout à fait comparables à ceux obtenus par Norris et Bukovac (1972) avec l'acide naphtalène-acétique (NAA). Avec le glyphosate, l'effet du $\mathrm{pH}$ est moins clair : il est plus faible sur la perméabilité que dans le cas du 2,4-DB, et dans nos conditions d'expérience il est nul sur la fixation. Gougler et Geiger (1981) ont observé l'absence d'effet du pH sur l'absorption cellulaire de cet herbicide. L'influence des cires cuticulaires solubles est plus réduite pour les deux herbicides testés que pour d'autres herbicides de type acide faible ou d'autres espèces cuticulaires (RIEderer et SCHONHERr, 1985 ; BaIllon, non publié). 
Ces résultats relatifs à la cuticule de feuille de gui, pourvue de nombreux ostioles, peuvent être comparés à ceux obtenus avec des cuticules issues de faces supérieures de feuilles sans stomates (CHAmel, 1986). Ceci suggère que le passage des herbicides pourrait se faire directement à travers la cuticule, et non par l'ostiole des stomates, comme semblaient déjà l'indiquer nos observations en microscopie électronique à balayage. Les cuticules isolées montrent, en effet, des ostioles fermés.

\subsection{Distribution dans le parasite}

Le transport des herbicides hors des feuilles traitées est sous la dépendance du stade de développement du gui :

- Appliqué bien avant le développement des jeunes pousses, l'herbicide se répartit dans l'ensemble du parasite (stade I, cas du glyphosate).

- Appliqué au début de la croissance, l'herbicide se concentre dans les jeunes pousses; le transport est préférentiellement acropète (stade II ; le stade I dans l'exemple du 2,4-DB étant un cas intermédiaire entre les stades I et II, puisque les jeunes pousses se sont développées à la fin des six semaines d'expérience).

- Appliqué en fin de croissance, l'herbicide est transporté dans les deux sens, basipète et acropète, mais il reste localisé sur un seul axe au niveau de chaque feuille traitée (stade III).

A tous les stades considérés, la radioactivité est présente dans le système endophytique à l'état de traces, excepté au stade III dans le cas du glyphosate, où il apparaît très marqué. Toutefois, ces résultats ne portent que sur six semaines d'expérience, or pour une efficacité maximale des herbicides sur le gui, il faut attendre 4 à 6 mois. Ceci permet de supposer que le transport de l'herbicide vers le système endophytique se poursuit au cours de cette longue période. De plus, dans le système endophytique, les cordons corticaux ont une activité mitotique permanente au cours de l'année, et celle des suçoirs augmente au tout début du printemps (SALLE, 1979). Le système endophytique constitue donc un centre d'appel pour les substances véhiculées par le phloème, mais de moindre importance comparé aux jeunes pousses (BAILlon, 1988). Par contre, la radioactivité n'a jamais été observée dans la branche-hôte. Ceci conforte les résultats précédents (Seledzhanu et Galan-Fabian, 1961 ; Balllon, 1985), suggérant l'absence de passage de substances carbonées du gui vers l'hôte.

L'étude du transport du saccharose, principale forme de transport des glucides dans la sève élaborée, a été réalisée parallèlement. Elle a permis de mettre en évidence des analogies avec la distribution des herbicides aux différents stades de développement du gui ; les jeunes pousses constituant les principaux centres d'appel (BaILlon et al., 1985 ; BAILlon, 1988). Ces résultats confirment donc que les deux herbicides sont exportés par le phloème hors de la feuille traitée (Sprankle et al., 1975a et 1975b ; Fernandez et BAYER, 1977 ; Fletcher et Kirkwood, 1982). De plus, l'importante migration de radioactivité observée avec le glyphosate, notamment vers les feuilles d'un an, peut être expliquée par les travaux de Wills (1978), Gougler et Geiger (1981) et Dewey et APPLEBY (1983). Ils suggèrent la possibilité d'une redistribution de cet herbicide dans la plante par le flux transpiratoire après le transport basipète des molécules et leur pénétration à travers la membrane des tubes criblés (herbicide ambimobile). Par contre le 2,4-D et le 2,4-DB, présents sous forme dissociée dans le liber ( $\mathrm{pH}$ alcalin), ne peuvent pas retraverser cette membrane (EL Ibaoui et al., 1986). D'autre part, pour les 
deux herbicides considérés, la migration hors des feuilles traitées diminue fortement en quantité au moment du développement des jeunes pousses (stade II). Ce résultat est surprenant comparé à l'augmentation de l'exportation du saccharose mise en évidence à ce stade (BAillon, 1988). Plusieurs hypothèses peuvent être avancées :

- immobilisation des molécules (herbicides ou produits de dégradation) sur des sites non actifs dans les tissus foliaires (FLETCHER et KIRKWOOD, 1982) ;

- apparition d'un effet phytotoxique des herbicides, qui empêcherait la prise en charge des molécules par le phloème.

Mais il ne faut pas exclure la possibilité d'artefact lié aux techniques d'étude utilisées : contrairement aux expérimentations effectuées avec les herbicides, l'étude du transport phloémien a été réalisée à l'aide de branches de pommier parasitées par le gui et placées en survie dans l'eau. Le fait de sectionner la branche-hôte peut modifier le potentiel hydrique du système hôte-parasite et intervenir sur le transport phloémien des assimilats.

Il faut souligner ici l'absence de données bibliographiques relatives à la migration des herbicides phloème-mobiles chez le gui. Seuls des travaux de LEonard et Hull (1965 et 1966) rapportent des résultats qualitatifs chez Phoradendron, un « gui » nordaméricain. Ces résultats sont d'ailleurs comparables à ceux que nous avons obtenus chez Viscum album.

\subsection{Essai d'efficacité}

Les observations confirment les résultats déjà obtenus par Frochot et al. (1983) quant à l'efficacité des herbicides. La lenteur de réponse du gui laisse supposer que les herbicides ne sont pas métabolisés entièrement au cours des six semaines d'expérience, mais il ne faut pas exclure la possibilité d'un effet dû à la lenteur de leurs déplacements ou de leurs actions physiologiques. Par ailleurs, nous ne pouvons pas conclure définitivement sur l'apparition de repousses après traitements. En effet, cet essai et d'autres expériences réalisées sur gui du peuplier (Balloon et Frochot, 1987) montrent que des repousses peuvent apparaître au bout de 2 à 3 ans, mais elles semblent moins fréquentes - aux concentrations efficaces - avec le glyphosate qu'avec les phytohormones de synthèse, herbicides auxquels se rattachent le 2,4-DB. Pour ces deux derniers points, il est nécessaire de poursuivre les observations et les expérimentations en vraie grandeur. D'autant plus que dans ces essais, les traitements ont été localisés sur les touffes de gui.

\section{Conclusion}

La cuticule de feuille de gui représente la première barrière que doivent franchir les substances exogènes pour pénétrer dans les tissus sous-jacents. C'est pourquoi nous avons envisagé l'étude de la pénétration cuticulaire (fixation et perméabilité). Les résultats obtenus à l'aide de cuticules isolées ont montré que le 2,4-DB peut traverser plus facilement la cuticule que le glyphosate. Toutefois, la vitesse de pénétration reste toujours très faible. La pénétration cuticulaire de ces deux herbicides est une fonction linéaire de la concentration. Par contre, l'augmentation du pH entraîne une diminution 
de la pénétration dans le cas du 2,4-DB, mais ne semble pas avoir d'effet sur celle du glyphosate. Les cires cuticulaires contribuent de façon importante à la faiblesse de la pénétration.

La distribution des herbicides dans le parasite a été suivie à trois stades différents du développement du gui. Elle présente des analogies avec celle du saccharose. L'herbicide tend à se concentrer dans les jeunes pousses qui constituent des centres d'appel. L'exportation des herbicides à partir des feuilles traitées est élevée (20 à 30 p. 100) juste avant l'apparition des jeunes pousses, elle devient très faible (1 à 4 p. 100) au moment de leur développement. Par ailleurs, quelque soit le stade phénologique étudié, la radioactivité est décelable en faible quantité dans le système endophytique du gui, six semaines après le dépôt foliaire des herbicides. L'étude des variations saisonnières du transport phloémien du saccharose a permis de montrer qu'une faible activité physiologique du gui persiste au cours de l'hiver, notamment le transport phloémien basipète (BAILlon, 1988). Les similitudes existant entre le transport des deux herbicides phloème-mobiles étudiés et celui des assimilats, ainsi que les essais d'efficacité de ces herbicides sur le gui, nous conduisent à confirmer l'intérêt d'un traitement hivernal en période de repos de l'hôte. A ce stade, les herbicides se répartissent dans l'ensemble du plant de gui et entraînent la destruction de sa partie aérienne au bout de quelques mois. Les faibles niveaux de radioactivité décelés dans le système endophytique ne permettent pas de préjuger de la durée d'efficacité, c'est-àdire de l'apparition ou non de rejets au cours des années qui suivent l'application. Des expérimentations de terrain à plus long terme sont nécessaires pour répondre à cette question (BAillon et Frochot, 1987).

L'absence de radioactivité dans la branche-hôte, six semaines après les applications aux différents stades, montre que les risques de phytotoxicité par passage de l'herbicide du gui à l'hôte sont nuls. Des applications localisées sur des touffes de gui peuvent être réalisées avec ces herbicides sans danger pour la plante-hôte. Par contre, des pulvérisations généralisées peuvent entraîner des dommages sur les arbres-hôtes par passage direct de l'herbicide à travers l'écorce. Il est nécessaire de définir avec précision les conditions de réalisation de ces traitements.

L'expérimentation au laboratoire a été conduite avec des matières actives pures, c'est-à-dire présentées différemment des spécialités commerciales. Cette différence ne joue probablement pas sur la distribution des herbicides dans la plante, ni sur les principes qui régissent leur pénétration à travers la cuticule. Par contre, la formulation de la spécialité commerciale, ainsi que la composition de la solution, interviennent pour favoriser la pénétration foliaire, notamment par la présence d'agents mouillants et d'adjuvants. Cette étude doit être encore élargie aux produits commerciaux pour aboutir à des règles pratiques d'utilisation et à une plus grande compréhension des phénomènes observés.

Reçu le 15 juin 1987.

Accepté le 17 septembre 1987.

\section{Remerciements}

Les auteurs tiennent à remercier tout spécialement MONSANTO S.A. pour la fourniture du ${ }^{14} \mathrm{C}$-glyphosate. 


\section{Summary}

\section{Mistletoe chemical control. Penetration, translocation, efficiency of two phloem-mobile} herbicides (2,4-DB and glyphosate)

A chemical method to control mistletoe (Viscum album album, BALL) is investigated with two phloem-mobile herbicides (2,4-DB and glyphosate), directly applied on the parasite leaves.

Before being translocated in the parasite, the herbicides must penetrate through the cuticle of mistletoe leaves (fig. 2). The influence on cuticular sorption and permeability of different parameters linked to the herbicide solution (concentration, $\mathrm{pH}$ ) or to the cuticle itself (cuticular waxes), is studied in vitro (isolated cuticles) with the two herbicides (tabl. 1 and 2). 2,4-DB penetrates more easily than glyphosate, even if always in low amount. The cuticular penetration is directly linked to the concentration. At the opposite, a $\mathrm{pH}$ increase leads to a decrease of 2,4-DB penetration, whereas no effect is observed with glyphosate. Moreover, the cuticular waxes largely contribute to the low penetration.

Then, the distribution of the herbicides is followed in the mistletoe, which is always fixed on its host, at three different phenological stages of the parasite. It confirms the analogy with those of the assimilates, i.e., the sink feature of the new growing shoots. The translocation depends on the phenological stage of mistletoe (fig. 3-tabl. 3) :

- Applied before young shoot development, the herbicides are distributed throughout the parasite (fig. 3B, stage 1).

- Applied at the beginning of new shoot growth, the herbicides concentrate in these young shoots (fig. 3A, stage I and fig. $3 \mathrm{~A}$ and $\mathrm{B}$, stage II).

- Applied at the end of the growth, the herbicides are translocated in two directions, basipetal and acropetal, but only on one axis from the treated leaf (fig. 3A and B, stage III).

More over, six weeks after the treatment, the herbicides are always detected in the endophytic system, even if in low amount, whereas no traces are detected in the host-tissue.

At last, the mistletoe response is quite different with the two herbicides: on one hand, the minimal efficient concentration is lower with $2,4-\mathrm{DB}$ than with glyphosate (10 against $40 \mathrm{~g}$ of active matter per liter). On the other hand, particular symptoms characterize each herbicide (tabl. 4). This leads to conclude that these herbicides may be used efficiently and without phytotoxicity for the host, when the applications are localized on the mistletoe plants. The early spring, before young shoot development, seems to be the most interesting period for a treatment.

Key words : Viscum album album, hemiparasite, symptoms of phytotoxicity, cuticular sorption, cuticular permeability.

\section{Références bibliographiques}

Baillon F., 1985. Etude physiologique du Gui des Feuillus (Viscum album L. album) pour la recherche d'une méthode de lutte chimique contre ce parasite : Pénétration et transport de deux herbicides phloème-mobiles $(2,4-D B$ et glyphosate). Thèse de Doctorat de l'Université de Grenoble, $103 \mathrm{p}$.

Balloon F., 1988. Seasonal variations of respiration, phloem-transport and carbohydrate content in european mistletoe. Plant Physiol. Biochem., 26 (1), 85-91.

Baillon F., Chamel A. et Fer A., 1983. Comportement du 2,4-DB et du Glyphosate marqués appliqués sur le gui. Pénétration, transport et redistribution dans le parasite. Proc. $12^{c}$ Conférence du COLUMA, Paris, 1, 149-156.

Baillon F., Fer A. et Chamel A., 1985. Etude physiologique du Gui des Feuillus (Viscum album album L.) pour la recherche dune méthode de lutte chimique contre ce parasite. Société Française de Physiologie Végétale, Nantes, $1^{\text {er }}$ juin.

Baillon F. et Frochot H., 1987. La lutte chimique contre le gui des feuillus. Pénétration et distribution dans le gui de deux herbicides à absorption foliaire. Phytoma, 391, 16-21. 
Ball P.W., 1964. Loranthaceae. Flora europea. Cambridge Univ. Prop., 1, 72-73.

Chamel A., 1986. Foliar absorption of herbicides : study of the cuticular penetration using isolated cuticles. Physiol. Vég., 24, 491-508.

Chamel A. et Bougie B., 1977. Absorption foliaire du cuivre. Etude de la fixation et de la pénétration cuticulaires. Physiol. Vég., 15, 679-693.

Chamel A. et Gambonnet B., 1982. Study with isolated cuticles of behaviour of zinc applied to leaves. J. of Plant Nutrition, 5, 153-171.

Delabraze P. et Lanier L., 1972. Contribution à la lutte chimique contre le gui (Viscum album L.). Eur. J. For. Pathol, 2, 95-103.

Deom P.. 1981. Le gui. La Hulotte, 48 et 49, 48 p. et 52 p.

Dewey S.A. et Appleby A.P.. 1983. A comparison between glyphotase and assimilates translocation patterns in tall morningglory (Ipomea purpurea). Weed Sci., 31, 308-314.

El Ibaoui H., Delrot S., Besson J. et Bonnemain J.L., 1986. Uptake and release of a phloemmobile (glyphosate) and a non-phloem-mobile xenobiotic by broadbean leaf tissues. Physiol. Vég., 24, 431-442.

Fernandez C.H. et Bayer D.E., 1977. Penetration, translocation anx toxicity of glyphosate in bermudagrass (Cynodon dactylon). Weed Sci., 25, 396-400.

FLETCHER W.W. et KirKwOod R.C., 1982. Herbicides and plant growth regulators. Ed. Granada, London, $408 \mathrm{p}$.

Frochot H. et Delabraze P., 1979. Efficacité d'herbicides du groupe des aryloxyacides sur le gui du sapin. Proc. $10^{\circ}$ Conférence du COLUMA, Paris, 2, 789-804.

Frochot H. et Sallé G., 1980. Modalités de dissémination et d'implantation du gui. R.F.F., $X X X I I, 6,505-519$.

Frochot H., Pitsch M. et Wehrlen L., 1983. Efficacité d’herbicides sur le gui des feuillus (Viscum album mali) installé sur le peuplier. Proc. $12^{\circ}$ Conférence du COLUMA, Paris, 1. 157-165.

Frochot H. et WEHRLEN L., à paraître. Individualisation par bouturage de branches de peuplier parasitées par le gui.

Gougler J.A. et Geiger D.R., 1981. Uptake and distribution of N-phosphono-methyl-glycine in sugar beet plants. Plant Physiol., 68, 668-672.

LÉonard O.A. and Hull R.J., 1965. Translocation relationships in and between mistletoes and their host (Arceuthobium, Phoradendron). Hilgardia, 37, 115-153.

Léonard O.A. and Hull R.J., 1966. Translocation of ${ }^{14} \mathrm{C}$-labelled substances and ${ }^{32} \mathrm{PO}_{4}$ in mistletoe-infected and uninfected conifers and dicotyledonous trces. Isotopes in Weed Research, 31-46.

Maréchal J., 1983. Action de l'acide naphtyl-1-acétique ou ANA sur le gui du peuplier (Viscum album L.). Proc. I ${ }^{\circ}$ Conférence du COLUMA, Versailles, 2, 355-358.

Norris R.F. et Bukovac M.J., 1972. Effect of $\mathrm{pH}$ on penetration of naphtaleneacetic acid and naphtaleneacetamide through isolated pear leaf cuticles. Plant Physiol., 49, 615-618.

Riederer M. et Schönherr J., 1985. Accumulation and transport of (2,4-dichlorophenoxy) acetic acid in plant cuticles. II. Permeability of the cuticular membrane. Ecotoxicology and Environmental Safety, 9, 196-208.

Sallé G., 1979. Le système endophytique du Viscum album: anatomie et fonctionnement des suçoirs secondaires. Can. J. Bot., 57, 435-449.

Seledzhanu N. and Galan-Fabian D., 1961. Nutrition of common mistletoe. Soviet Plant Physiol., 8, 436-442.

Sprankle P., Megitt N.F. et Penner D., 1975a. Absorption, mobility and microbial degradation of glyphosate. Weed Sci., 23, 229-234.

Stranki.e P., Megitt N.F. et Penner D., 1975b. Absorption, action and translocation of glyphosate. Weed Sci., 23, 235-240.

Von Tubeuf K., 1923. Monographie der Mistel., Oldenbourg R., München and Berlin, 835 p.

WILLs G.D., 1978. Factors affecting toxicity and translocation of glyphosate in cotton (Gossypium hirsutum). Weed Sci., 26, 509-513. 